*Correspondence: e-mail: tegirish5@yahoo.co.in

,Received: .201 holes

\section{Introduction}

\title{
On the Cosmological Origin of the Rest Mass of Elementary Particles: The Ideas of Maximons and Minimons Revisited
}

\author{
Vikraman Vipindas ${ }^{1}$, Trivandrum Easwaraiyer Girish ${ }^{1 *}$ Chellappan Radhakrishnan Nair \\ Department of Physics, University College, Trivandrum, 695034, India
}

- $\quad$ Accepted/Published Online: .201

- $\quad$ Final Version: ..201

\begin{abstract}
:
It is suggested that physical properties of common elementary particles can be associated with microscopic Primordial Black Holes (PBH) which is inferred to have formed between $10^{-24}$ to $10^{-20}$ seconds from Big bang in the early universe. This is also found to be related to the phenomenon of Hawking radiation from these PBH. We have revisited the properties of minimons and maximons introduced by Markov [1] in this context. Planck particles which is inferred to form near Planck time $\left(3.857 \times 10^{-43}\right.$ seconds) are identified as maximons with a mass $\sqrt{\pi} m_{p}$ where $m_{p}$ is the Planck mass. The minimons are associated with a $\mathrm{PBH}$ with Hawking temperature identical with the cosmic microwave background temperature of the universe. The mass of the minimons are found to be comparable to that of the lightest neutrinos $(0.0185 \mathrm{eV})$. They also possess highest Compton wavelength $\left(10^{-4} \mathrm{~m}\right)$ known for an elementary particle.
\end{abstract}

Keywords: elementary particles; cosmological origin; maximon; Hawking radiation; neutrino mass; primordial black

The origin of the elementary particle mass spectrum remains still a puzzle. In this context the proposal of limits to the rest masses of elementary particles by Russian Academician Markov becomes relevant [1]. He suggested that elementary particles with maximum rest mass (maximons) are possibly Planck mass black holes formed in early universe. Further the particles with minimum rest mass (minimons) are likely to be related to neutrinos. Since maximons are supposed to have a cosmological origin related to dark matter [2] it will be important to investigate possible associations between matter in present universe and the physical constituents of early universe like primordial black holes (PBH). Quantum mechanics, Quantum gravity and super-Planck scale physics may play an important role in this context. The early universe lack direct astrophysical observations and is described only by standard cosmological models [3]. The physical concepts related to the formation of micro black holes (black holes whose size is comparable to that of elementary particles) took shape in 1970's [4] Prediction of Hawking radiation from black holes is a major development in this context [5] which unifies Classical thermodynamics, General relativity and Quantum mechanics as a special case. Recently there is a revived interest in the physics of $\mathrm{PBH}$ and Hawking radiation from purely theoretical physics point of view.

In this paper we have proposed a heuristic theory for the cosmological origin of the rest mass of elementary 
VIPINDAS, GIRISH, RADHAKRISHNAN NAIR

1 particles. This is done by associating rest mass of elementary particles with gravitational coupling constant and 2 certain physical properties of primordial black holes formed in very early universe. The ideas of maximons and minimons are revisited in this context explaining their possible physical significance.

\section{Maximons and the Graviational coupling constants of elementary particles}

5 Fine structure constant defines the strength of electromagnetic forces between the elementary particles. In a 6 similar way the constant which defines the strength of gravitational forces between the elementary particles is called gravitational coupling constant. It was defined originally for electrons by Feynman [6] and later this 3 definition is extended to other particles like protons [7]. We have generalized these definitions so that it is applicable for all elementary particles. The gravitational coupling constant $\alpha_{G}$ of an elementary particle of rest mass $m$ is defined as

$$
\alpha_{G}=\frac{2 \pi G m^{2}}{h c}=\left[\frac{m}{m_{p}}\right]^{2}
$$

Here $G, h$ and $c$ are fundamental constants and $m_{p}$ is plank mass.

From (1) we can find that

$$
m=\sqrt{\frac{h c \alpha_{G}}{G}}
$$

According to (2), the rest mass of an elementary particle is defined to be proportional to the square root of the quantum of the gravitational coupling constant.

Markov's expression for maximons [1] can be written as

$$
m_{\max }=\sqrt{\frac{h c\left(\frac{\alpha}{2}\right)}{G}}
$$

If we put $\alpha=1$ in (3) we find that $m_{\max }<m_{p}$. So the expression for maximons as suggested by Markov needs a correction.

If $\alpha=\alpha_{G}$ then equations (2) and (3) looks similar but not identical. When $\alpha_{G}=1$ we can find from (2) that the rest mass of the particle becomes

$$
m=\sqrt{\frac{h c}{G}}=m_{p}
$$

Here $m_{p}$ is the Planck mass, the value of mass suggested for maximons by Markov. So our expression seem to be a better relation to find rest mass of maximons compared to that of Markov.

If $\alpha_{G}=\pi$ then we can find from (2) that

$$
m=\sqrt{\frac{h c}{2 G}}=\sqrt{\pi} m_{p}
$$

We propose that (5) may be a new value for the mass of maximons whose cosmological origin and physical significance will be discussed in the following sections. 
VIPINDAS, GIRISH, RADHAKRISHNAN NAIR

3. Physical associations between elementary particles and Primordial Black holes formed in the early universe

3 The mass-radius relation of a Schwarzschild black holes given by

$$
R_{s}=\frac{2 G M}{c^{2}}
$$

4 Let

$$
R_{s}=\frac{h}{m c}=\lambda_{c}
$$

${ }_{5}$ Here $\lambda_{c}$ is the Compton wavelength and $m$ is the rest mass of the elementary particles associated with the 6 Schwarzschild black holes.

From (6) and (7) we can find that

$$
M=\frac{h c}{2 G m}
$$

8 Primordial black holes satisfying (7) and (8) can be defined as 'quantised' PBH hereafter. Considering values 9 of minimum and maximum rest mass of elementary particles we can find the value of $M$ in (3) will lie between 10 $4.72 \times 10^{11} \mathrm{~kg}$ (approx $10^{14} \mathrm{~g}$ ) to $1.64 \times 10^{15} \mathrm{~kg}$ (approx $10^{18} \mathrm{~g}$ ) which is equal to the rest mass of tau particles 11 and electrons respectively.

Carr equation [8] connecting mass $(M)$ and time $t$ of formation ( $t$ is reckoned from the Big Bang) of ${ }_{13} \mathrm{PBH}$ in the early universe is given by

$$
M(t)=10^{15}\left(t / 10^{-23}\right) \mathrm{g}
$$

14 or

$$
M(t)=10^{35} t \mathrm{~kg}
$$

Scharwzhild radius of a primordial black hole for the above elementary particle is

$$
R_{s}=\frac{2 G M}{c^{2}}=\lambda_{c}
$$

${ }_{21}$ Where

$$
M=\frac{h c}{2 G m}
$$

22 Let $m_{p p}$ be the mass of Planck particle, where

$$
m_{p p}=\sqrt{\frac{h c}{2 G}}
$$




\section{VIPINDAS, GIRISH, RADHAKRISHNAN NAIR}

$$
m_{p p}=\sqrt{\pi} m_{p}
$$

2 where $m_{p}$ is the plank mass.

We have

$$
m_{p p}^{2}=\pi m_{p}^{2}
$$

Equation (13) can be written as

$$
\begin{aligned}
& M=\frac{\pi m_{p}^{2}}{m} \\
& \frac{m}{M}=\frac{\alpha_{G}}{\pi}
\end{aligned}
$$

${ }_{6} \quad$ Mass density of black hole mass $M$ is $\rho_{b}=\frac{M}{V}$, where $V=\frac{4 \pi R_{s}^{3}}{3}$. Since $R_{s}=\frac{2 G M}{c^{2}}$ we get

$$
\rho_{b}=\frac{3 c^{6}}{32 \pi G^{3} M^{2}}
$$

7 For a Planck mass black hole the density will be identical to plank density

$$
\rho_{p l}=\frac{3 c^{6}}{32 \pi G^{3} m_{p l}^{2}}
$$

8

$$
\frac{\rho_{b}}{\rho_{p l}}=\frac{m_{p l}^{2}}{M^{2}}
$$

$9 \quad$ Using (17) and (18)

$$
\pi \frac{\rho_{b}}{\rho_{p l}}=\frac{\alpha_{G}}{\pi}
$$

10 The Scwarchild radius $\left(r_{s}\right)$ of an elementary particle is defined as

$$
r_{s}=\frac{2 G m}{c^{2}}
$$

11 The Scwarchild radius of $\mathrm{PBH}\left(R_{s}\right)$ with quantum characteristics discussed in Section 3 is

$$
R_{s}=\frac{2 G M}{c^{2}}
$$

12 Dividing (23) by (24) we get

$$
\frac{r_{s}}{\lambda_{c}}=\frac{m}{M}
$$

${ }_{13}$ Now we can write a master relation for PBH with quantum characteristics

$$
\frac{r_{s}}{\lambda_{c}}=\frac{m}{M}=\pi \frac{\rho_{b}}{\rho_{p l}}=\frac{\alpha_{G}}{\pi}
$$




\section{VIPINDAS, GIRISH, RADHAKRISHNAN NAIR}

1

2

\section{Planck particles as maximons}

Planck particles are hypothetical particles whose Compton wavelength and Schwarchild radius are identical [9] They are elementary particles as well as black holes.

Putting $r_{s}=\lambda_{c}$ in the master equation derived for PBH with quantum characteristics we can find that

$$
m / M=1 \text { or } m=M
$$

Also $\alpha_{G}=\pi$

Substituting this in (27) we can find that

$$
M=m=\sqrt{\frac{h c}{2 G}}=\sqrt{\pi} m_{p}
$$

This is identical to equation (5).

Using Carr equation [10] we can find that a PBH with Planck particle mass will form at time $t$ from big bang in the very early universe so that

$$
t=3.857 \times 10^{-43} \text { seconds }
$$

Thus we can find that Planck particles are elementary particles with maximum rest mass. They are maximons which form close to but later than Planck time in the very early universe.

\section{Hawking radiation and minimons}

The Hawking temperature $\left(T_{h}\right)$ of a $\mathrm{PBH}$ is defined as

$$
T_{h}=\frac{h c^{3}}{16 \pi^{2} k_{B} G M}
$$

The energy $\left(E_{h}\right)$ of Hawking radiation from the $\mathrm{PBH}$ is

$$
E_{h}=k_{B} T_{h}
$$

Here $k_{B}$ is the Boltzmann constant [10].

From eqn (8),

$$
M=\frac{h c}{2 G m}
$$

Therefore

$$
\begin{gathered}
T_{h} k_{B}=\frac{m c^{2}}{8 \pi^{2}} \\
E_{h}=\frac{m c^{2}}{8 \pi^{2}}=\frac{E_{r}}{8 \pi^{2}}
\end{gathered}
$$

Here $E_{r}$ is rest mass energy of the elementary particle associated with Hawking radiation pair production. Let minimum value of Hawking temperature (30) be equal to CMBR temperature $T_{h}(\min )$

$$
T_{h}(\min )=2.725 \mathrm{~K}
$$


VIPINDAS, GIRISH, RADHAKRISHNAN NAIR

$1 \quad$ Hawking radiation energy

$$
E_{h}(\min )=k_{B} T_{h}(\min )
$$

2 Value of Boltzmann constant

$$
k_{B}=1.38 \times 10^{-23} \text { units }(\mathrm{SI})
$$

3

From above equations we can find

$$
E_{h}(\min )=2.347 \times 10^{-4} \mathrm{eV}
$$

From (34) we can find rest-mass energy of elementary particle associated with the Hawking radiation is

$$
m c^{2}(\min )=8 \pi^{2} E_{h}(\min )
$$

Substituting value of $E_{h}(\min )$ from $(38)$

$$
m c^{2}(\min )=0.0185 \mathrm{eV}=0.02 \mathrm{eV}(\text { approx })
$$

So the minimum rest mass energy of an elementary particle is $10^{-2} \mathrm{eV}$.

In comparison with the recent inferences of the mass of neutrino [11-13] rest mass of the particle given in eqn (40) is suggested to be the minimum absolute mass of neutrino.

The relation $\frac{m}{M}=\frac{\alpha_{G}}{\pi}$ can be related to the pair production phenomena associated with Hawking radiation from $\mathrm{PBH}$ in very early universe.

The mass of the particle involved in the Hawking radiation pair production $(m)$ is proportional to the gravitational force of attraction between the pairs of such particles in the black hole.

\section{Discussion and Conclusions}

In this paper we have found a new expression for the rest mass of elementary particles involving the gravitational coupling constants $\left(\alpha_{G}\right)$ of these particles. This expression is found to be more accurate than a similar one proposed for maximons by Markov [1]. The coupling constant suggested by Markov $(\alpha)$ is identified as $\alpha_{G}$ in this work. Markov suggested that maximons are black holes with Planck mass. In our theory on the cosmological origin of elementary particles [14] we suggest that every elementary particle is associated with a unique primordial black hole (with mass $M$ ) formed in early universe. We have also found that $m / M$ is proportional to the gravitational coupling constant of the elementary particle $\left(\alpha_{G}\right)$ associated with the PBH. This association is explained in terms of the pair production phenomena associated with hawking radiation from these particles. In this context we have revisited the ideas of maximons and minimons first proposed by Markov [1]. Planck particles are proposed by us as maximons. Further the elementary particle associated with a PBH with Hawking temperature identical to that of cosmic microwave background temperature is proposed by us as minimon which is likely to be neutrinos with least mass [15].

Density perturbations are considered to be one of mechanisms suggested for the formation of $\mathrm{PBH}$ in the early universe [16]. In the master relation found by us for $\mathrm{PBH}$ with quantum characteristics the term $\left(\rho_{b} / \rho_{p l}\right)$ can be considered as the amplitude of the density fluctuations in cosmic vacuum. The Planck density $\rho_{p l}$ is equivalent to the vacuum density [17]. For the maximons the amplitude of the primordial density fluctuations 


\section{VIPINDAS, GIRISH, RADHAKRISHNAN NAIR}

1 related to the formation of associated PBH will be maximum. In our theory for Planck particles we find that

$$
\frac{\rho_{b}}{\rho_{p l}}(\text { maximon })=1 / \pi
$$

2 This implies that the density of Planck particle black hole is only approximately one third of the Planck density. 3 For Planck mass particles $\left(\rho_{b} / \rho_{p l}\right)=1$, is it physically justifiable?

$4 \quad$ The value for minimons where the amplitude of density perturbations is minimum we find

$$
\frac{\rho_{b}}{\rho_{p l}}(\text { minimon })=0.89 \times 10^{-61}
$$

${ }_{5} \quad$ The value for the mass (in SI units) for minimons in our paper is

$$
m(\text { minimon })=2.05 \times 10^{-38} \mathrm{~kg}
$$

6 The $\mathrm{PBH}$ associated with minimon inferred to have a mass of $0.73 \times 10^{23} \mathrm{~kg}$ which has probably formed close to the end of electro-weak era in early universe with inferred time of $0.73 \times 10^{-12}$ seconds from Big Bang as estimated from Carr equation.

The mass of minimon inferred in this paper $\left(10^{-2} \mathrm{eV}\right)$ is of the same order as some estimates of neutrino mass reported in literature [11-13]. It is worth noting that the Compton wavelength of minimon is a maxima.The corresponding value for minimon in this work is found to be approximately $10^{-4} \mathrm{~m}$ or $100 \mu \mathrm{m}$. This value is perhaps highest reported for an elementary particle. Quantum effects are experimentally detected up to a size of $40 \mu \mathrm{m}$ [18]. It may be interesting to note that some bio molecules (eg. DNA of fruit fly etc) which may have quantum characteristics [19] has a size much greater than that of the Compton wave length of minimons inferred in this paper.

\section{References}

[1] Markov MA. Journal of Experimental and Theoretical Physics Letters 1987; 45 (3): 141-144.

[2] Markov MA. Physics Letters A 1993; 172 (5): 331-332.

[3] Weinberg S. Gravitation and Cosmology: Principles and Applications of the General Theory of Relativity. Wiley India Ltd, Reprint, 2011.

[4] Hawking S. Monthly Notices of Royal Astronomical Society 1971; 152 (1): 75-78.

[5] Hawking S. Communications in Mathematical Physics 1975; 43 (3): 199-220.

[6] Feynman RP. Acta Physica Polonica A 1963; 24 (5): 697-722.

[7] Keifer C. Why quantum Gravity? Stamatescu and E. Seiler (Editors) Approaches to Fundamental Physics, Lecture Notes in Physics 721: 123-130 Berlin, Heidelberg: Springer, 2007 DOI: 10.1007/978-3-540-71117-9.

[8] Carr BJ. Primordial Black Holes as a Probe of Cosmology and High Energy Physics. In: Giulini DJW, Kiefer C, Lmmerzahl C. (editors) Quantum Gravity. Lecture Notes in Physics, 631 Berlin: Springer, 2003.

[9] Crowthers SJ, Dunning-Davies J. Progess in Physics 2006; 3 (1): 70-73.

[10] Kawabera H. Hawking radiation of black holes, www.kiso.phys.se.tmu.ac.jp/thesis/m.h.kuwabara.pdf 2014.

[11] Gando A, Gando Y, Hachiya T, Hayashi A, Hayashida S et al. (KamLAND-Zen Collaboration), Phys. Rev. Lett. 2016; 117 (8): 109903. 


\section{VIPINDAS, GIRISH, RADHAKRISHNAN NAIR}

[12] Penedo JT, Petcov ST. Physics Letters B, 2018; 786 (1): 410-417.

[13] Bilensky SM, Pascoli S, Petcov ST. Preprint: arXiv:hep-ph/0102265v3 2012.

[14] Girish TE. Evidence for high density early universe in elementary particle physical data, Proceedings of the International conference on the advances in astroparticle physics and cosmology, Saha Institute of Nuclear Physics, India, available in IAEA/INIS, Ref. No. 48057022, 48 (27), 2015.

[15] Vipindas V, Girish TE, Radhakrishnan Nair C. Probing of the quantum nature of primordial black holes through Hawking radiation and estimating the lower limit to the mass of neutrino, Proceedings of the National Conference on Cosmology and Particle Physics (NC-CAPP 2018), Women's Christian College, Chennai, 2018.

[16] Green AM. Primordial black holes - sirens of early universe, in Quantum Aspects of Black Holes, Fundamental theories of Physics, Calmet (editor) Springer Publication, 2015, pp. 129-149.

[17] Yousef MH. Duality of time: Complex time geometry and perpetual creation of space, UAE: UAE University, 2018, pp. 158.

[18] O'Connel AD, Hofheinz M, Ansmann M, Radoslaw C Bialcazk, Lenander M et al. Nature 2010; 464 (1): $697-703$.

[19] Troels Koch, Irene Shim, Morten Lindow, Henrik Ørum, and Henrik G. Bohr Nucleic acid therapeutics, 24, DOI: 10.1089/nat.2013.04652014. 